\title{
A New Method for Fracturing Wells Reservoir Evaluation in Fractured Gas Reservoir
}

\author{
Jianchun Guo, Yong Xiao, and Haiyan Zhu \\ State Key Laboratory on Oil and Gas Reservoir Geology and Exploitation, Southwest Petroleum University, Chengdu 610500, China \\ Correspondence should be addressed to Yong Xiao; xiaoyongwork@163.com
}

Received 7 January 2014; Revised 2 March 2014; Accepted 4 March 2014; Published 10 April 2014

Academic Editor: Sarp Adali

Copyright (C) 2014 Jianchun Guo et al. This is an open access article distributed under the Creative Commons Attribution License, which permits unrestricted use, distribution, and reproduction in any medium, provided the original work is properly cited.

\begin{abstract}
Natural fracture is a geological phenomenon widely distributed in tight formation, and fractured gas reservoir stimulation effect mainly depends on the communication of natural fractures. Therefore it is necessary to carry out the evaluation of this reservoir and to find out the optimal natural fractures development wells. By analyzing the interactions and nonlinear relationships of the parameters, it establishes three-level index system of reservoir evaluation and proposes a new method for gas well reservoir evaluation model in fractured gas reservoir on the basis of fuzzy logic theory and multilevel gray correlation. For this method, the Gaussian membership functions to quantify the degree of every factor in the decision-making system and the multilevel gray relation to determine the weight of each parameter on stimulation effect. Finally through fuzzy arithmetic operator between multilevel weights and fuzzy evaluation matrix, score, rank, the reservoir quality, and predicted production will be gotten. Result of this new method shows that the evaluation of the production coincidence rate reaches $80 \%$, which provides a new way for fractured gas reservoir evaluation.
\end{abstract}

\section{Introduction}

Natural fracture is one of the most important migration channels and accumulation spaces in fractured gas reservoirs. In order to obtain good fracturing treatment in this kind of gas reservoir, the first is to develop natural fracture characterization study and make clear the characteristics of the natural fracture system and the parameters impacting fracture development. Narr [1] measured the fracture-spacing index by density of joints, and Hanks et al. [2] stated that lithology is the primary control and structural position playing a greater role in the overall fracture character and density. Huang et al. [3] used the mud-loss data to characterize natural fracture and Norbeck et al. [4] stated that several well testing methods such as acoustic and resistivity image logs are available to the industry to identify natural fractures.

In order to obtain stimulation effect successfully, an accurate mathematical model is needed to describe the nonlinear relationship of various factors and to set up evaluation index system and decision-making system for reservoir evaluation after making clear the natural fracture development characteristics. Economides and Martin [5] categorized the reservoir evaluation for candidate-well selection methodologies into three types: (1) conventional, (2) mixed conventional and advanced, and (3) advanced methods; Zoveidavianpoor et al. [6] stated that fuzzy system model predicts and ranks wells accurately.

In the paper, the author takes member 2 of Xujiahe formation of Hechuan fractured gas reservoir as an example to establish the parameter index system representing the degree of natural fracture development on the basis of tectonic characteristics, core observation, lithology characteristics, and drilling and logging display data. Meanwhile, he analyzes the nonlinear relationship of various factors affecting the stimulation effect, sets up evaluation index system and decision-making system, and then completes the quantitative reservoir evaluation of fracturing well.

\section{Gas Reservoir Geology Background and Natural Fracture Characterization}

2.1. Gas Reservoir Geology Background. Hechuan gas field is an NE axial trend minor axis anticline-like structure, located 

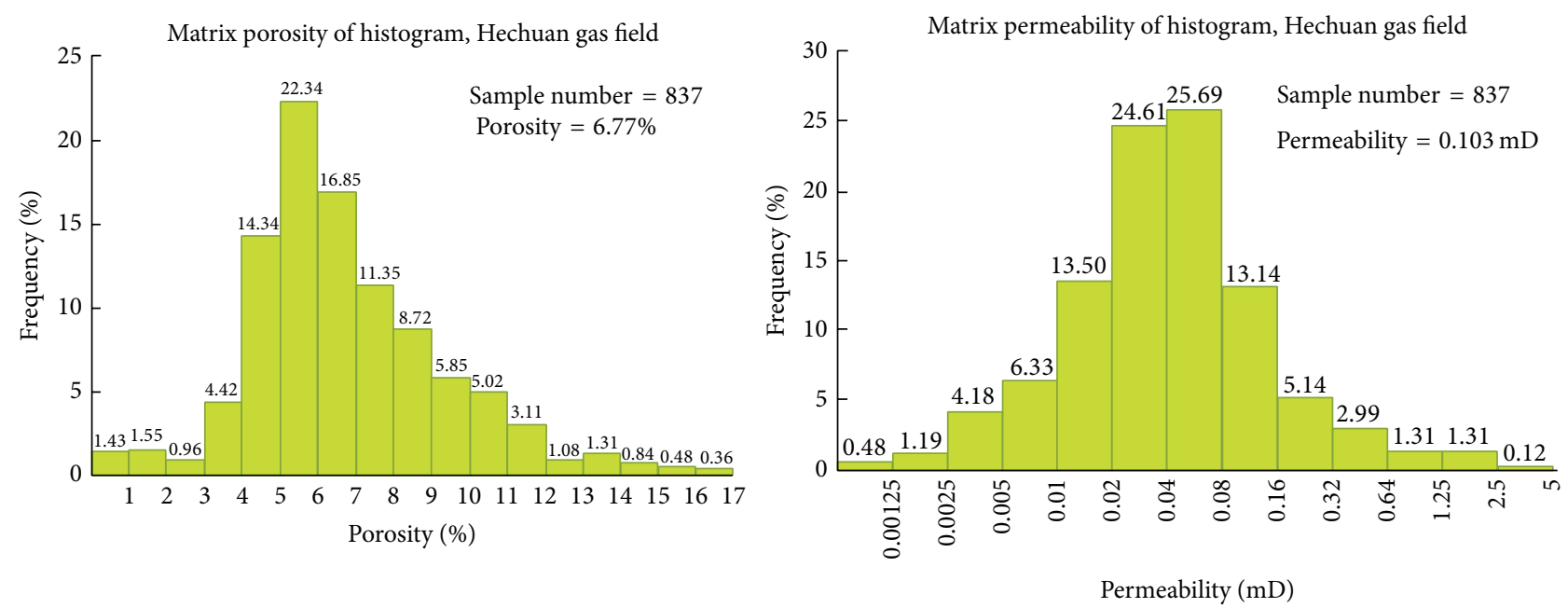

FIGURE 1: Porosity and permeability distribution in Hechuan gas field.

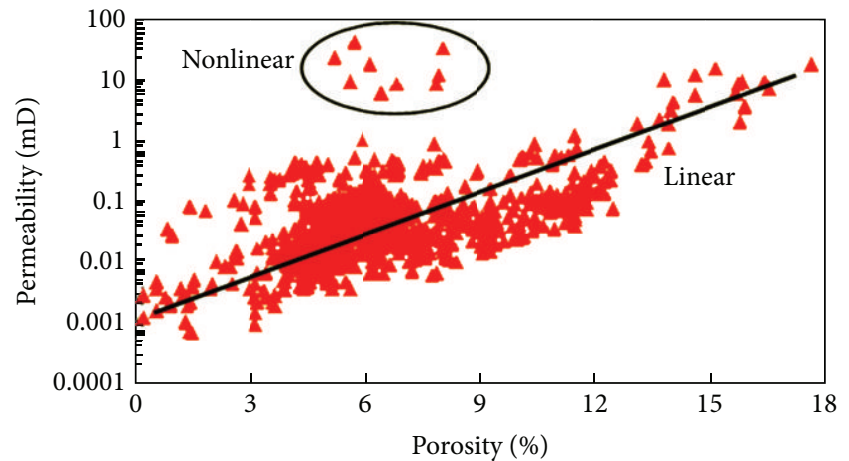

FIGURE 2: Relationship between porosity and permeability in Hechuan gas field.

in the east-central of the gentle structure of the Sichuan basin, which will produce microcracks easily under tectonic stress. The results of 720 slice samples show that the lithology is gray medium grained and medium-fine grained lithic arkose, feldspathic lithic sandstone, and lithic quartz sandstone mainly. Reservoir space types are mainly intergranular pores, intragranular dissolved pore, mixed pore and microfractures, and so forth.

According to the analysis of 837 pieces' reservoir core sample, the porosity distribution is from $0.2 \%$ to $17 \%$, and the average porosity is $6.77 \%$; the permeability is concentrated in $0.001 \mathrm{mD}$ to $3.114 \mathrm{mD}$ mainly, and the average permeability is $0.103 \mathrm{mD}$. This study is shown in Figure 1 schematically. Figure 2 shows a good linear relation existing between log permeability and porosity, but there is still some deviation, which proves that the reservoir has some natural fracture. Figure 3 shows an example of the core observation in member 2 of Xujiahe formation in Hechuan gas field; the result shows that high angle shear fractures with different cutting depth are often associated with quartz and calcite crystal.
2.2. Natural Fracture Characterization. By analyzing the field outcrop, core, and logging data comprehensively, the region's natural fracture development degree can be characterized by tectonic characteristics, core observation, drilling and logging display, and so on.

Tectonic Characteristics. Many researchers believe that different fold structure developed different structural curvature and stress concentration leading to different degree of fracture development and stimulation performance $[2,7-$ 9]. Figure 4 shows the relationship between test production and structural position in Hechuan gas field. Maximum stimulation performance is $6.53 \times 10^{4} \mathrm{~m}^{3} / \mathrm{d}$ when the gas well purpose layer is located in the top structure followed by northeast wing structure and northwest wing structure on $5.23 \times 10^{4} \mathrm{~m}^{3} / \mathrm{d}$ and $3.91 \times 10^{4} \mathrm{~m}^{3} / \mathrm{d}$, respectively, and the worst is the southern only on $1.11 \times 10^{4} \mathrm{~m}^{3} / \mathrm{d}$.

Lithologic Characteristics. Many scholars believe that the macro appearance such as mineral content, grain size, texture, and porosity can affect the mechanical properties of the rock and reflect the degree of the natural fracture development indirectly. The smaller rock particles make greater strength and density to produce cracks in smaller stress conditions $[2,7,10,11]$. Figure 5 shows that it will get different average stimulation test production with different reservoir lithology gas well, and the fine sandstone had the highest stimulation production at $4.75 \times 10^{4} \mathrm{~m}^{3} / \mathrm{d}$; hence fine sandstone may contain most natural fractures.

Logging Characteristics. Current imaging logging used for fracture identification and reservoir evaluation includes formation of microresistivity scanning imaging logging, azimuthal resistivity imaging logging, ultrasonic imaging well logging, dipole shear wave imaging logging, and nuclear magnetic resonance (NMR) imaging logging [12]. Based on the conventional logging response mechanism of the crack, the conventional well logging curve can be used to describe 


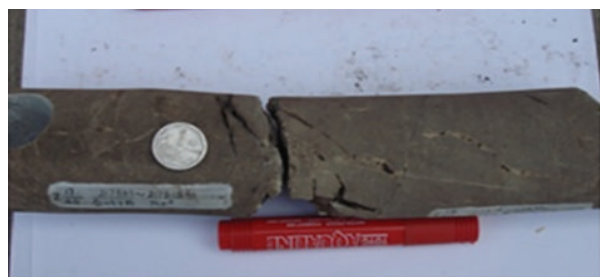

Different filling degree of high angle shear fractures, Hechuan 7 wells, $2179.2 \mathrm{~m}$

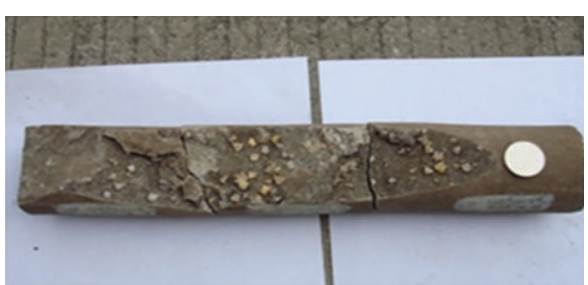

Anthigenic quartz calcite grain filling cracks, Hechuan 5 wells, $2278.9 \mathrm{~m}$

Figure 3: Core description in Hechuan gas field.

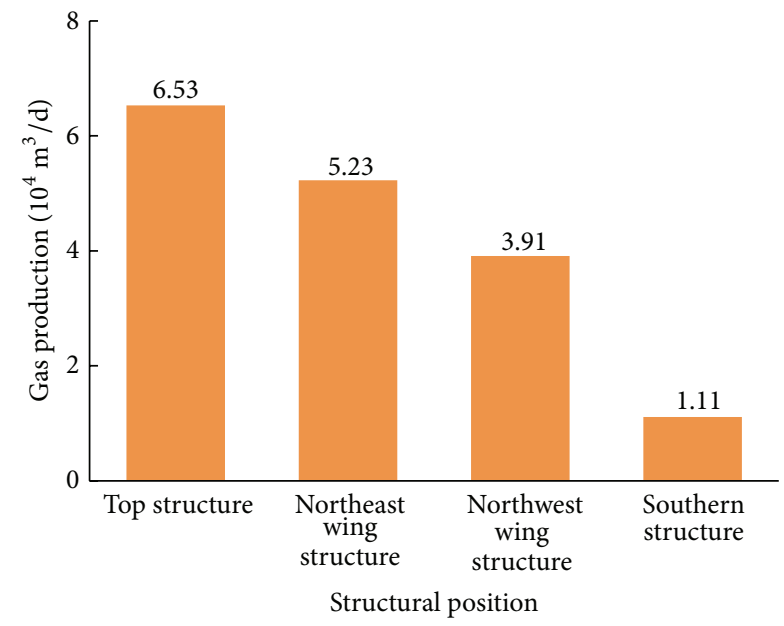

FIGURE 4: The relationship between test production and structural position in Hechuan gas field.

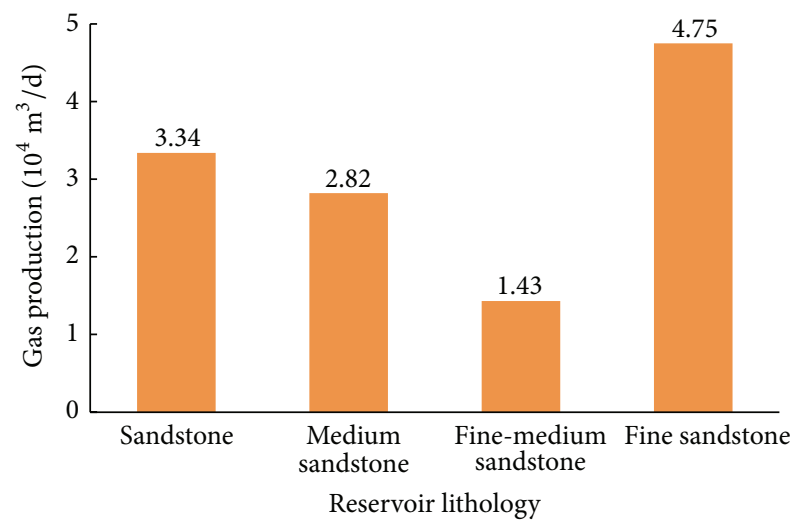

FIGURE 5: The relationship between test production and formation lithology in Hechuan gas field.

the number and distribution of the natural fractures (Dyke and $\mathrm{Wu}$ et al., 1995) [13]. By Figure 6, the imaging logging test results prove that the natural fracture development in this field is heterogenetic. Table 1 describes the different angles of natural fractures anomalies appearing in the logging.

Drilling and Logging Characteristics. The process of drilling mud leakage and the decrease of drilling time indicate that

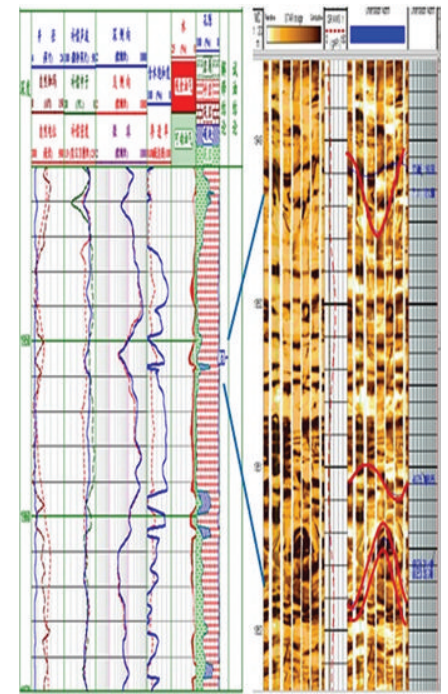

Figure 6: Imaging logging feature map of GN 2 well in Hechuan gas field.

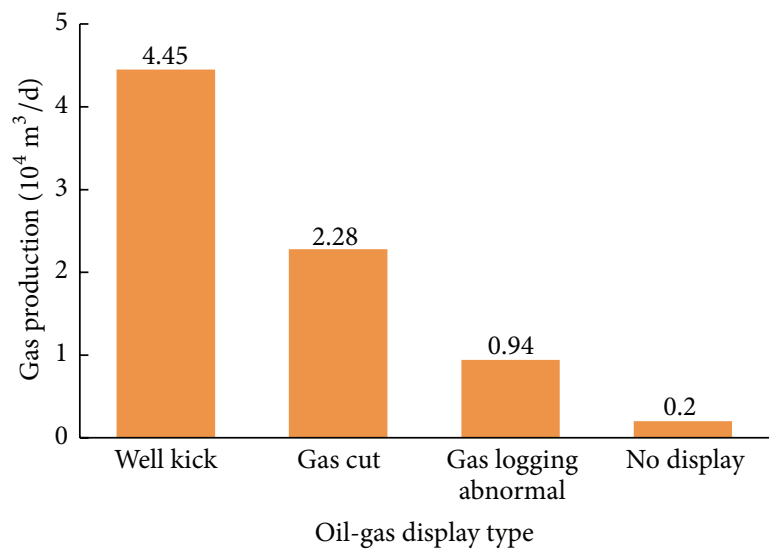

FIGURE 7: The relationship between test production and oil-gas display type in Hechuan gas field.

there is natural fractures development in this unconsolidated sandstone reservoir [14]. Figure 7 shows the different average stimulation test production with the different oil-gas display type, and the well kick has the highest stimulation production 
TABLE 1: The comprehensive comparison of various logging information.

\begin{tabular}{lll}
\hline Logging information & Low angle fracture & High angle fracture \\
\hline Full waveform logging & $\begin{array}{l}\text { Longitudinal and transverse wave energy attenuation } \\
\text { and shear wave attenuation are greater }\end{array}$ & $\begin{array}{l}\text { Longitudinal wave energy has an obvious } \\
\text { attenuation and subsequent wave } \\
\text { interference }\end{array}$ \\
Acoustic variable density log & $\begin{array}{l}\text { Longitudinal and transverse wave grey scale stripe } \\
\text { becomes shallow, appearing as herringbone } \\
\text { interference stripe }\end{array}$ & $\begin{array}{l}\text { Interference fringes appear in the } \\
\text { longitudinal and transverse wave }\end{array}$ \\
Dual laterolog & $\begin{array}{l}\text { Appears as low resistivity and negative difference in the } \\
\text { range of } 10 \text { to two thousand }\end{array}$ & $\begin{array}{l}\text { Resistivity appears as positive anomalies } \\
\text { about below the surrounding rock and it } \\
\text { is around hundreds of ohm meters }\end{array}$ \\
Acoustic logging & $\begin{array}{l}\text { Appears as high sonic differential time and cyclical } \\
\text { jumping phenomenon } \\
\text { Not shown obviously }\end{array}$ & $\begin{array}{l}\text { Not shown obviously } \\
\text { Dual laterolog }\end{array}$ \\
Lithodensity log & $\Delta \rho$ peak anomaly and increase if there is barite mud & $\begin{array}{l}\text { Sometimes it appears as rough borehole } \\
P_{e} \text { increase is not obvious with mud barite } \\
\text { presence }\end{array}$ \\
\hline
\end{tabular}

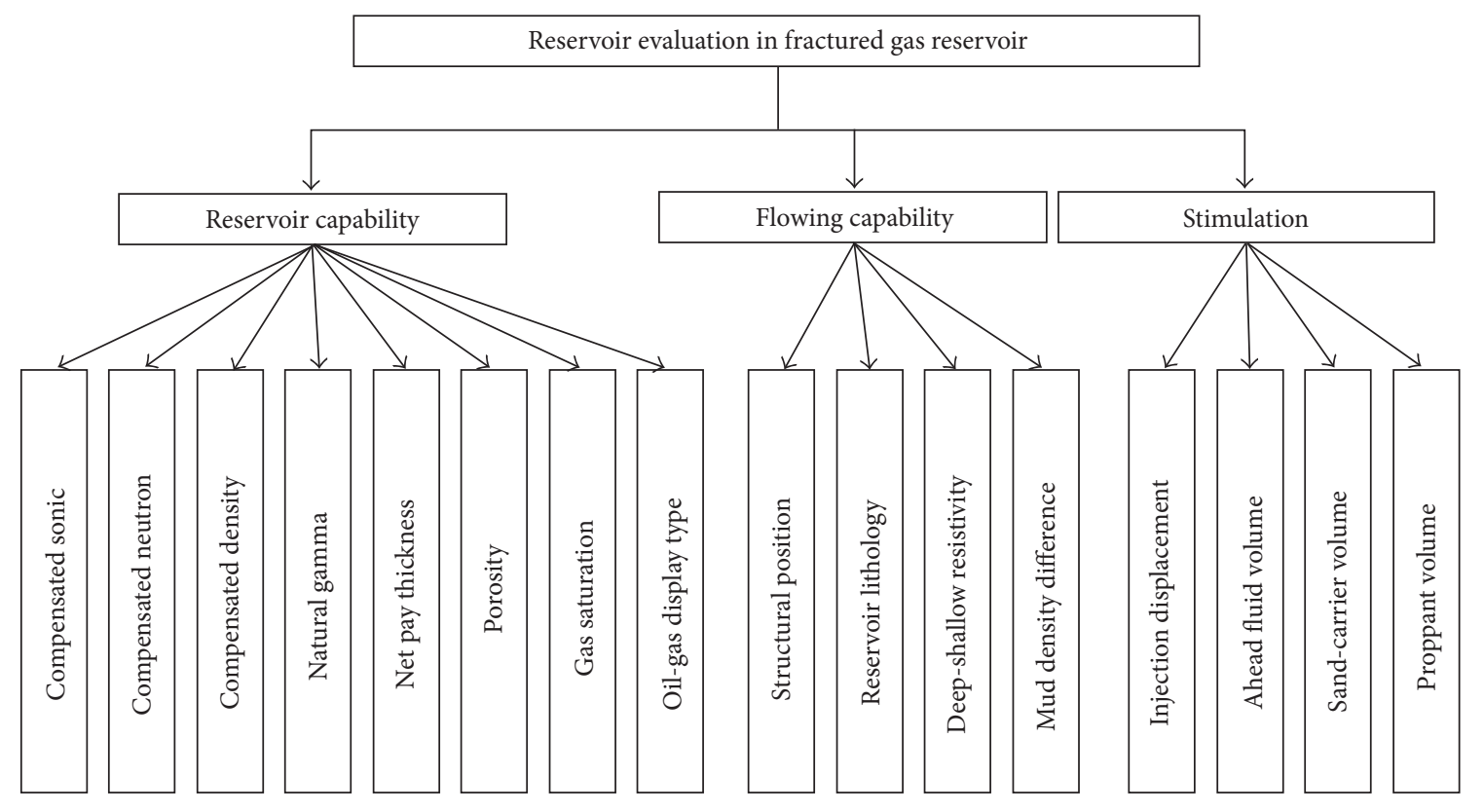

FIGURE 8: Comprehensive index evaluation system of fractured gas reservoir.

at $4.45 \times 10^{4} \mathrm{~m}^{3} / \mathrm{d}$ followed by gas cut and gas logging abnormal on $2.28 \times 10^{4} \mathrm{~m}^{3} / \mathrm{d}$ and $0.94 \times 10^{4} \mathrm{~m}^{3} / \mathrm{d}$, respectively, and there is almost no production when there is no oil-gas display in the process of drilling and logging.

\section{Fuzzy Logic and Multiple Grey Correlation}

3.1. Evaluation Index System. Through understanding the natural fracture gas reservoir and combining the analysis of drilling, logging, geological, and fracturing operation, the parameters which influence the fracturing effect are divided into three levels according to the secondary evaluation index system: the measurement of the reservoir capacity, flowing capability, and stimulation, as shown in Figure 8. Parameters characterize the following eight reservoir capabilities: compensated sonic, compensated neutron, compensated density, natural gamma, net pay thickness, porosity, gas saturation, and oil and gas display type; parameters characterize the following four capabilities: structural position, reservoir lithology, deep and shallow resistivity difference, and mud density difference; parameters characterize the following four kinds of stimulation: injection displacement, ahead fluid volume, sand-carrier volume, and proppant volume.

3.2. Fuzzy Logic. Many scholars have done a lot of research on reservoir evaluation method [15-18]. In this paper, the author, in view of the present public literature statistics, finds that the present reservoir evaluation methods mainly include four types, as shown in Figure 9.

Fitting function method extracts reservoir parameters and model of linear or nonlinear fitting function; it is simple and easy to calculate this model, but the requirement of 


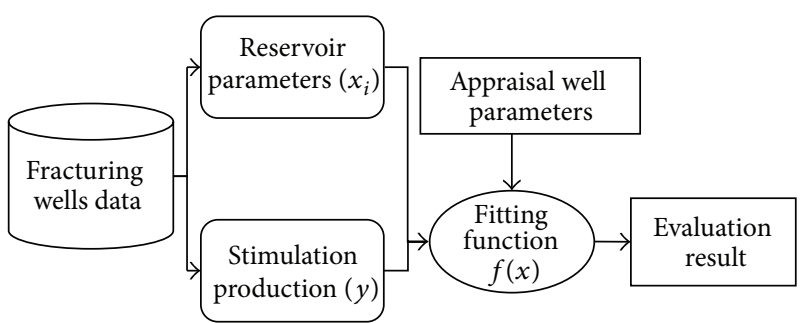

(a) Fitting function method

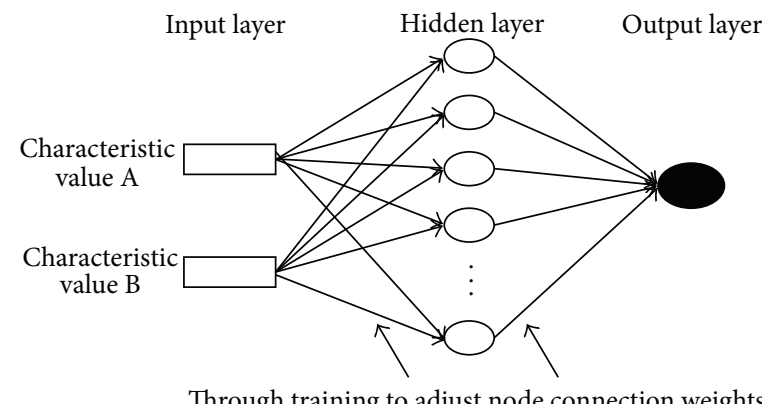

(c) BP neural network method

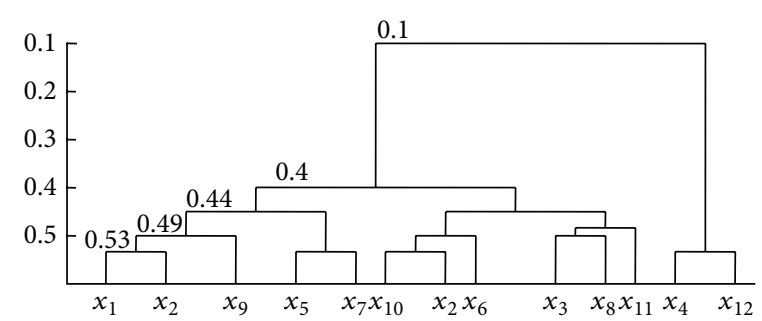

(b) Fuzzy clustering method

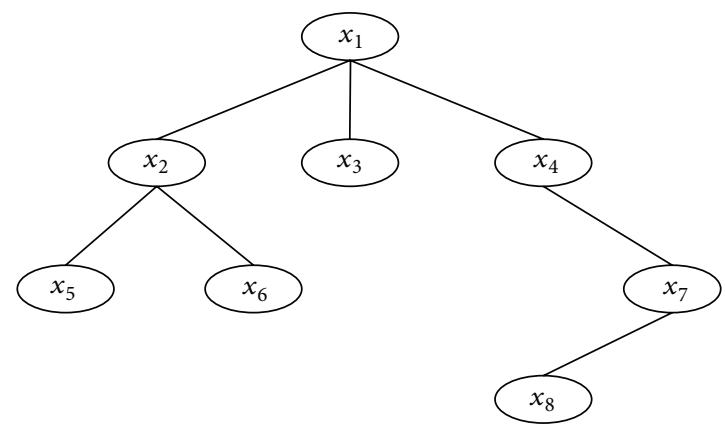

(d) C4.5 method

FIGURE 9: Comprehensive comparison of various reservoir evaluation methods.

independent parameters and calculation accuracy is poor (Figure 9(a)). Fuzzy clustering method establishing the fuzzy similarity matrix and the matrix elements can be grouped by intercept, but it is not easy to guarantee the precision (Figure 9(b)). The BP neural network gets the inherent relationship between the inputs and outputs and solves the involved problem by adjusting the weight of the neural network on line, and if there is new data to join you then it will need to adjust the network and recalculate again (Figure 9(c)). C4.5 method is to optimize the decision tree by adjusting the node properties, and the result is stable, reliable, and speedy, but it is difficult to adjust after constructing the decision tree (Figure 9(d)).

In conclusion, it is easy to model the fitting function and fuzzy clustering methods and computation speed is fast, but the nonlinear relationship of variables is not considered and the result is not accurate; $\mathrm{BP}$ neural network has some disadvantages such as running into local minimum value easily and slow convergence rate and C4.5 is difficult to adjust the construction of the decision tree. Therefore any single mathematical method is hard to describe the particularity of fractured gas well reservoir evaluation. According to the above research, the author proposes a new reservoir evaluation method based on fuzzy logic and multiple grey correlation.

Without the loss of generality, the principle of fuzzy logic evaluation usually supposes that there are $m$ factors affecting the effect of single well stimulation performance, which are called factors set $U$, at the same time reservoir geology is divided into $n$ different levels, which are called decisionmaking system set $V$. According to the principle of fuzzy logic evaluation, two datasets fields can be gotten as given by

$$
\begin{aligned}
& U=\left\{u_{1}, u_{2}, \ldots, u_{m}\right\}, \\
& V=\left\{v_{1}, v_{2}, \ldots, v_{n}\right\} .
\end{aligned}
$$

Take reservoir parameters as an example; the factors affecting stimulation effect are $u_{1}=$ porosity, $u_{2}=$ permeability, and $u_{3}=$ net pay thickness.et, so the evaluation factors set $U=\left\{\right.$ porosity, permeability, net pay thickness, $\left.\ldots, u_{m}\right\}$. Comments containing 4-5 grade can be expressed as $v_{1}=\mathrm{I}$ = Optimum, $v_{2}=\mathrm{II}=$ Better, $v_{3}=\mathrm{III}=$ Possible, $v_{4}=\mathrm{IV}=$ None, and the decision-making system set $V=\{$ I, II, III, IV $\}$. Therefore, each parameter $u_{i}$ in every comment $v_{i}$ has a membership degree $r_{i j}$, as shown by

$$
r_{i}=\left\{r_{i 1}, r_{i 2}, \ldots, r_{i n}\right\} .
$$

Membership degree matrix $R=\left(r_{i j}\right)_{m \times n}$ can be set up through using the membership function of single factor evaluation. And because the role of various factors in the decisionmaking system is not the same, then it defines the weight set $W, W_{i}$ representing the influence extent of each parameter to the stimulation performance:

$$
W=\left\{w_{1}, w_{2}, \ldots, w_{m}\right\}
$$

When the fuzzy weight vector $W$ and membership degree matrix $R$ are known, the gray correlation matrix $B$ is obtained 
TABLE 2: The major function used in fuzzy logic presented by Zoveidavianpoor et al. [6].
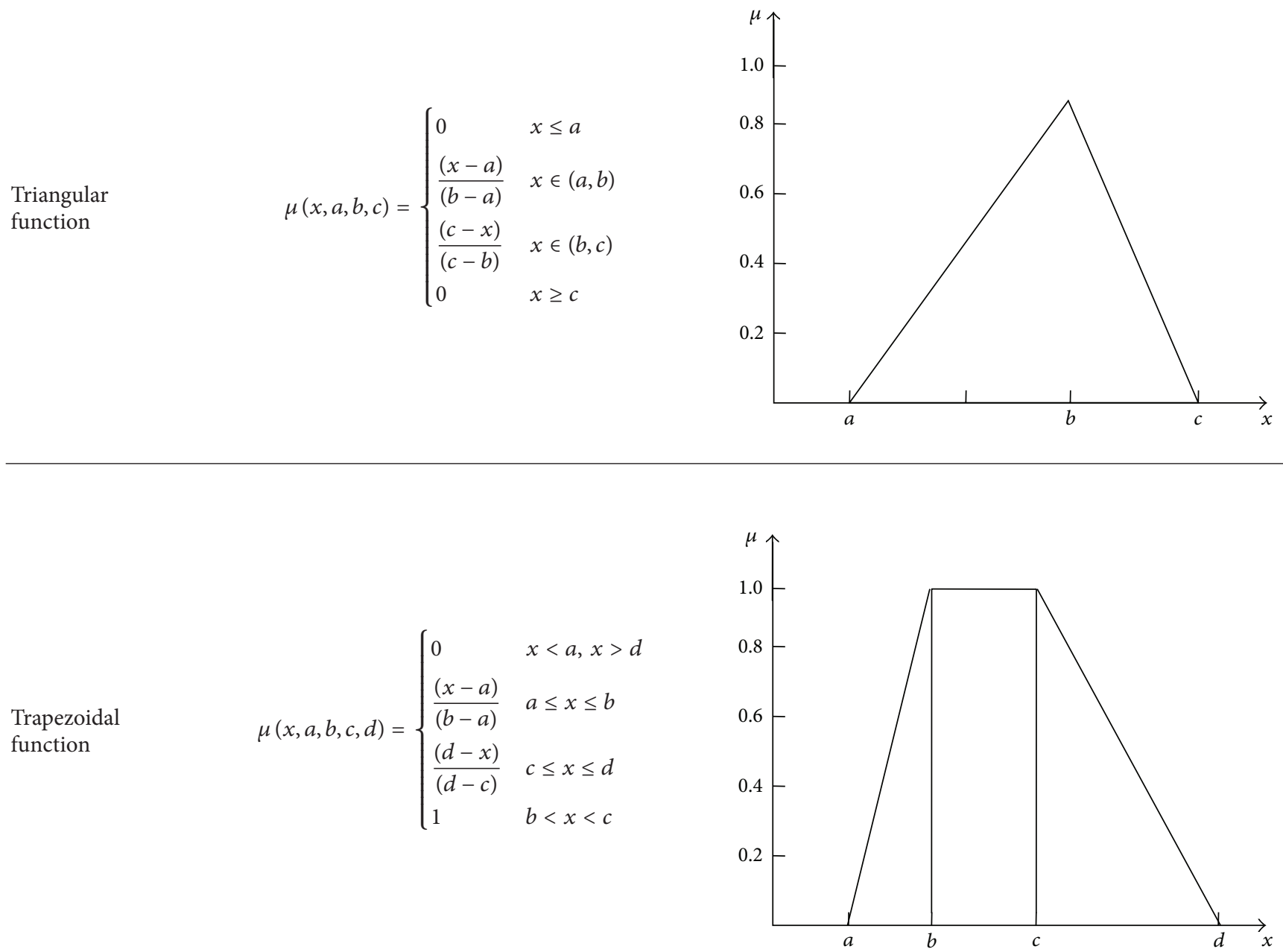

Normalized

Gaussian function

$$
\mu\left(x, \sigma_{i}, c_{i}\right)=\exp ^{\left(x-c_{i}\right)^{2} / 2 \sigma_{i}^{2}}
$$

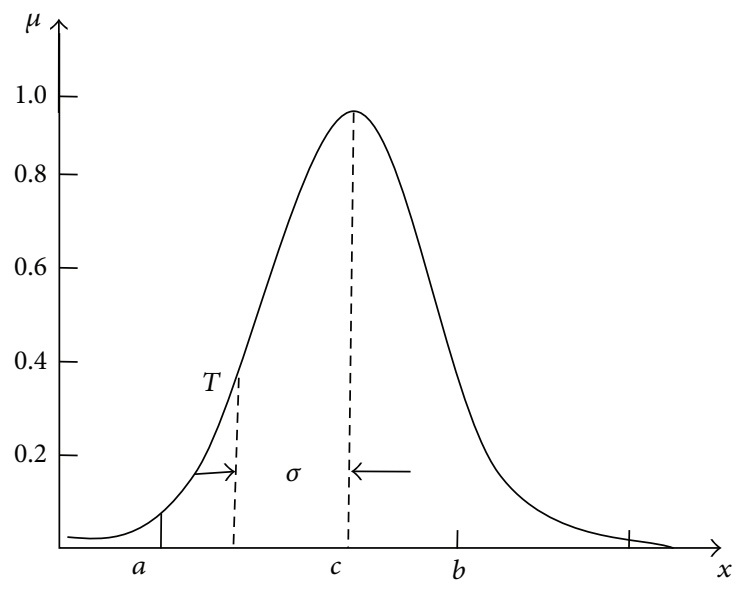


TABLE 2: Continued.

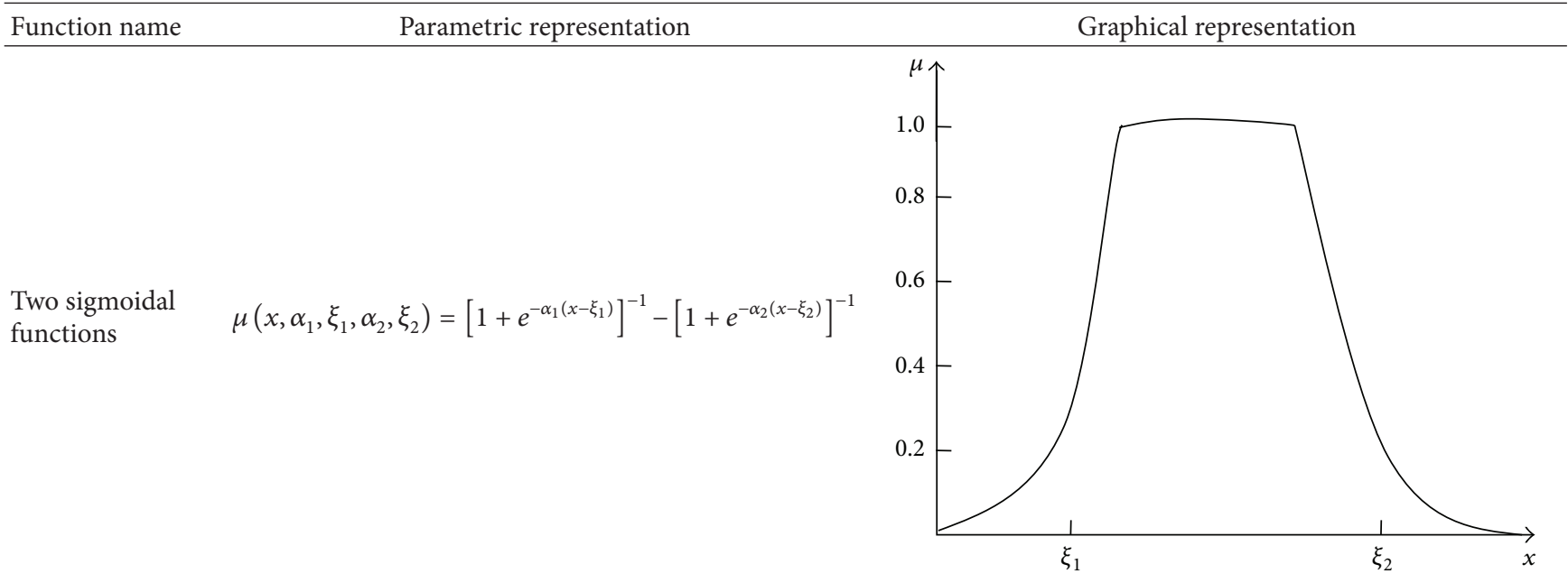

Generalized bell function

$$
\mu\left(x, \alpha_{i}, \beta, \xi\right)=\left[1+\left|\frac{x-\xi}{\alpha}\right|^{2 \beta}\right]^{-1}
$$

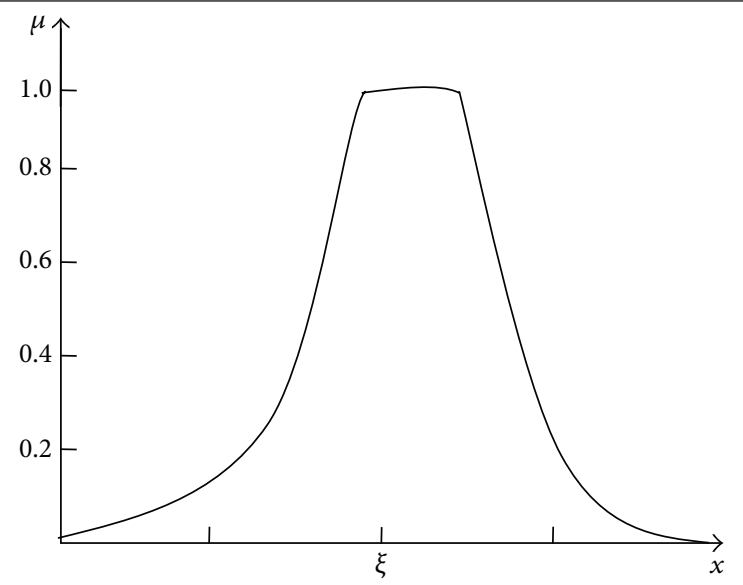

by fuzzy arithmetic operator; the following function demonstrates the operation process:

$$
B=W \cdot R=\left(b_{1}, b_{2}, \ldots, b_{n}\right) .
$$

The decision-making system value of every level is created artificially, such as $v_{1}=\mathrm{I}=100, v_{2}=\mathrm{II}=75, v_{3}=\mathrm{III}=50$, and $v_{4}=\mathrm{IV}=25$ :

$$
x=\frac{\sum_{j=1}^{m}\left(b_{j} \times v_{j}\right)}{\sum_{j=1}^{m} b_{j}} .
$$

3.3. Membership Function. Membership degree matrix $R$ is used to represent each of the parameters in the close degree of each comment level calculated by membership function. To determine the membership degree, the most important is to find accurate membership function. Fuzzy linear programming is proposed by Zimmermann [19]; they think the usual fuzzy programming problem can be described by simple linear, such as trapezoidal and triangular membership functions. But it is a problem for complex fuzzy decision, because a simple membership function is difficult to describe the nonlinear and multiple parameters events in reservoir evaluation accurately. Therefore, piecewise linear membership function was proposed by Narasimhan [20], but linear curve is not smooth and it is hard to characterize subordinate relations. Zoveidavianpoor et al. [6] summarized the main use of the membership function of fuzzy logic, shown in Table 2. Fractured gas reservoir evaluation is a multiparameter nonlinear problem; so the improved Gaussian model [21] is used to calculate the fuzzy sets, as shown in the following equation and Figure 10:

$$
\mu(x, c, \delta)=e^{-[(x-c) / \delta]^{2}}
$$

In the distribution of the Gussian membership function is generally believed if a given fuzzy interval of $x$, then the middle of the range value is $c$ and $\mu(c, c, \delta)=1$. Equal points in the adjacent comment grade are called the transition point; the transition point is the lower limit of previous evaluation level and the upper limit of below level, so the transition point 


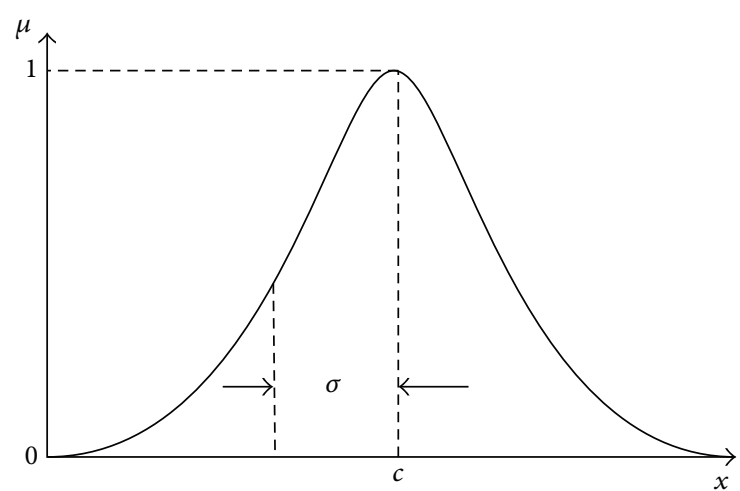

FIGURE 10: Gaussian membership function.

is 0.5 ; the following accurately described the mathematical relationship:

$$
\begin{gathered}
c=\frac{x_{1}+x_{2}}{2}, \\
e^{-\left[\left(x_{1}-x_{2}\right) / 2 \delta\right]^{2}}=0.5, \\
\delta=\frac{d_{1}-d_{2}}{1.66} .
\end{gathered}
$$

3.4. Multilevel Grey Correlation Analysis Method. Julong [22] presented the theory of grey correlation system; he stated that curve geometric similarity can be used to determine the contact tightness; if the curve similarity is high, so the greater degree of correlation between data sequence and the smaller the opposite. In this paper, the author studies that the problem of reservoir evaluation is a multi-index system, and the decision-making system is multilayered; then he uses the multilevel grey correlation evaluation weights for each level of parameters.

3.4.1. Single-Level Grey Correlation. First, you need to deal with each parameter standardization and dimension. Set $r_{0}$ for target sequence, and then you can calculate the $\xi_{i}(j)$, representing the correlation coefficient of $r_{i}$ in the first $j$ parameter, which is given by

$$
\xi_{i}(j)=\frac{\min _{i} \min _{j} \Delta_{i}(j)+\alpha \max _{i} \max _{j} \Delta_{i}(j)}{\Delta_{i}(j)+\alpha \max _{i} \max _{j} \Delta_{i}(j)},
$$

where $\alpha$ is distinguishing coefficient, $\alpha=0.5, \Delta_{i}(j)=\mid r_{o}(j)-$ $r_{i}(j) \mid$, and $c_{i}$ is correlation degree, which is given by

$$
c_{i}=\frac{1}{N} \sum_{j=1}^{n} \zeta_{o i}(j)
$$

Finally the weight set of each factor is obtained on the target by correlation degree, which is given by

$$
W_{i}=\frac{c_{i}}{\sum_{j=1}^{m} c_{i}} \text {. }
$$

3.4.2. Multilevel Grey Correlation. Multilevel grey correlation analysis process is similar to single-level analysis method. The factor set $U$ in the decision-making system can be divided into multilevel evaluation index. Take each level of parameters as a single-level comprehensive evaluation system, calculate the correlation coefficient of each parameters belongs to the secondary evaluation index. In this way each level correlation coefficient in decision-making system can be calculated, and the final correlation degree from the correlation of each level is gotten; it can be divided into three steps.

(1) Factors set $U$ can be divided into several subsets $u_{i}$, where $u_{i} \in U, i=1,2, \ldots, m$.

(2) Utility of single-level gray correlation function calculates the secondary level weight vector $W$, which is given by

$$
w=\left\{w_{1}, w_{2}, \ldots, w_{m}\right\}, \quad \sum_{i=1}^{p} w_{i}=1, \quad w_{i}>0 .
$$

(3) Membership degree matrix $R$ can be calculated through the fuzzy arithmetic operators between $u_{i}$ and weight vector $W$. The greater the $b_{j}$ is as close to its decision-making evaluation, the format is as follows:

$$
\begin{gathered}
B=w R=\left(b_{1}, b_{2}, \ldots, b_{n}\right), \\
B=\left[\begin{array}{c}
b_{1} \\
b_{2} \\
\vdots \\
b_{p}
\end{array}\right]=\left[\begin{array}{cccc}
b_{11} & b_{12} & \cdots & b_{1 n} \\
b_{21} & b_{22} & \cdots & b_{2 n} \\
\vdots & \vdots & \vdots & \vdots \\
b_{p 1} & b_{p 2} & \cdots & b_{p n}
\end{array}\right] .
\end{gathered}
$$

\section{Case Study}

Known from the analysis of the first section, member 2 of Xujiahe formation is a fractured gas reservoir in Hechuan gas field. In this paper, 15 fractured wells from this fractured gas reservoir are a sample database for reservoir evaluation. According to the characterization of natural fractures and the description of the geological characteristics in section one 16 parameters which affect stimulation effect are selected as the factors set $U$, number 16 being the target for reservoir evaluation in this study; all the parameters and data are shown in Table 3.

4.1. Establish Decision System. Establishing decision system, firstly, the parameter features need to be considered. Decision system for continuity parameters can be established from a lot of fractured well data by correlation analysis and average method, and discrete parameters without quantitative indicators fuzzy language is more suitable for this decision system. 


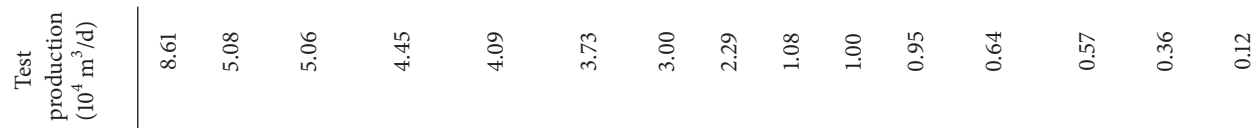

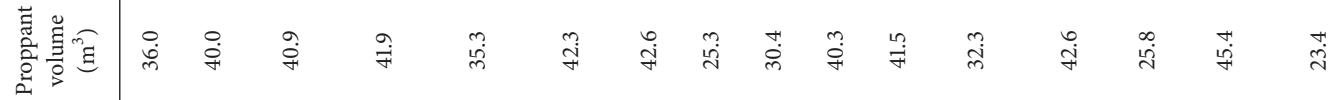

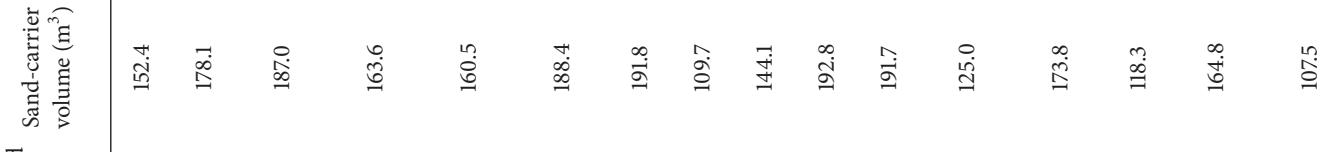

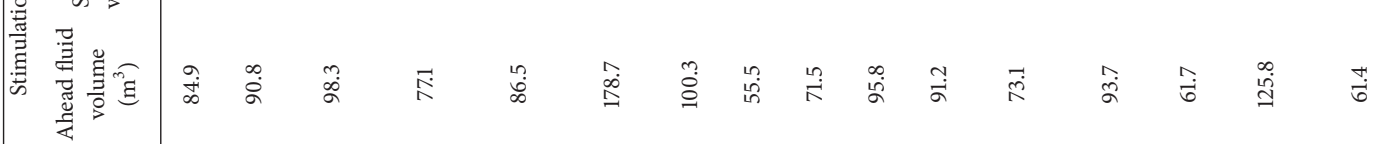

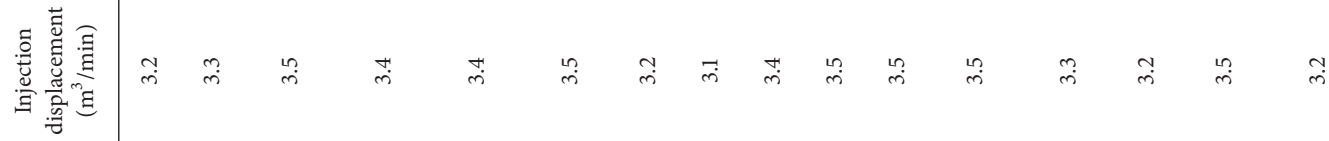

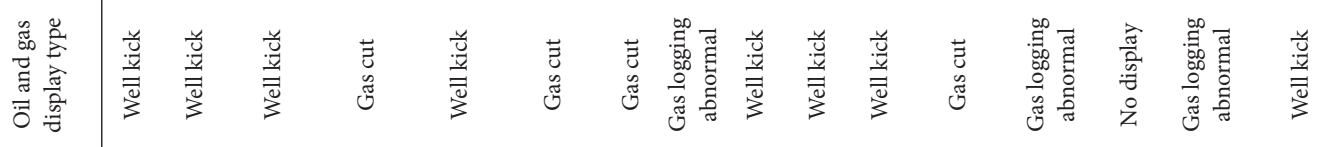

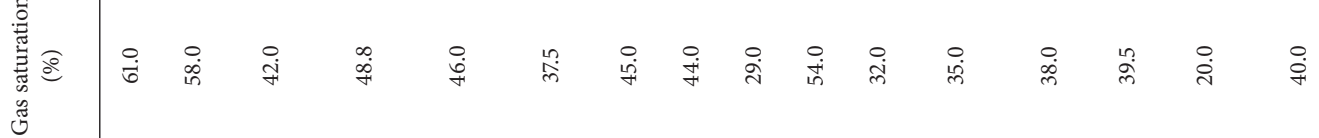

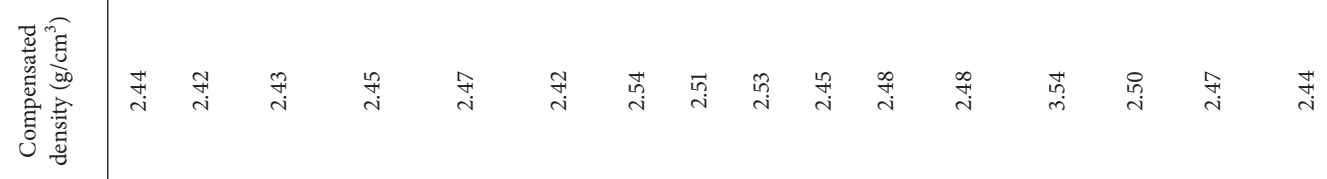

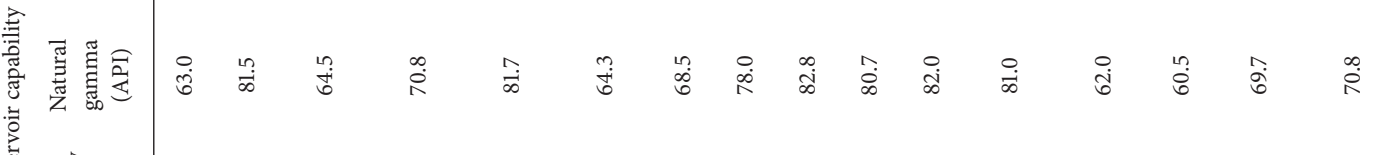

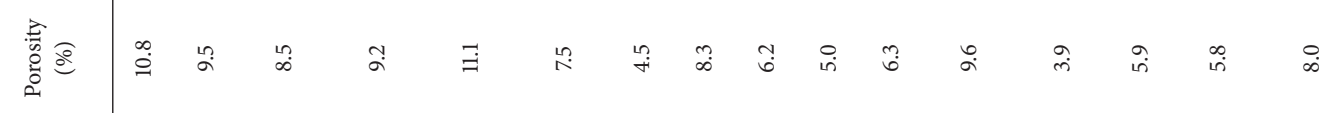

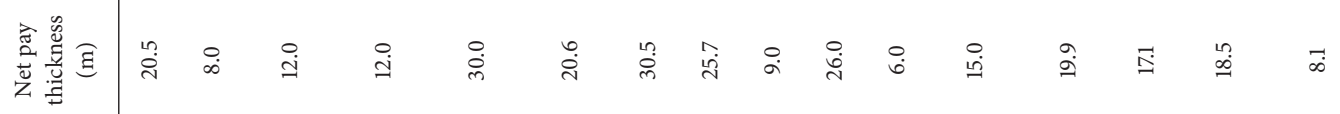

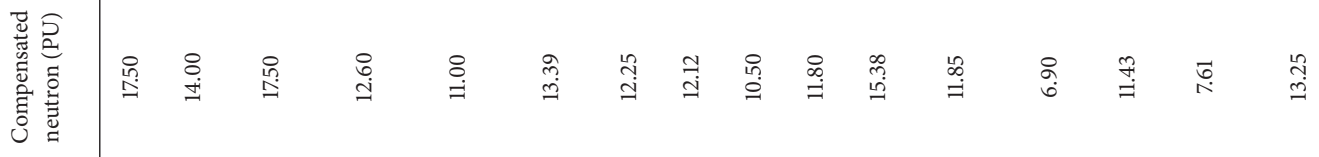

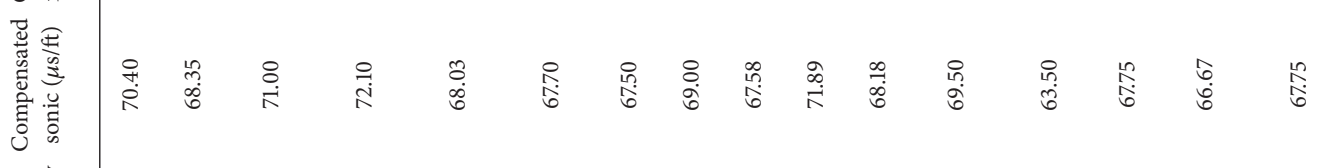

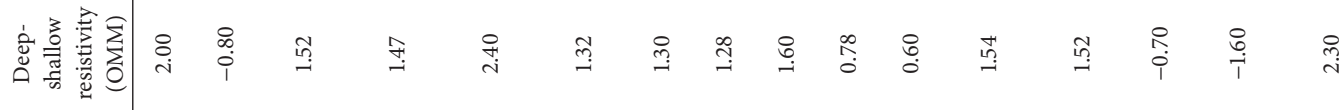

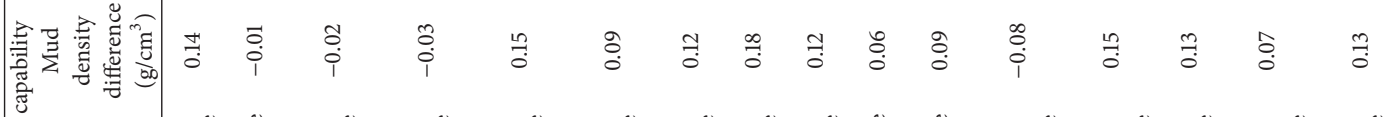

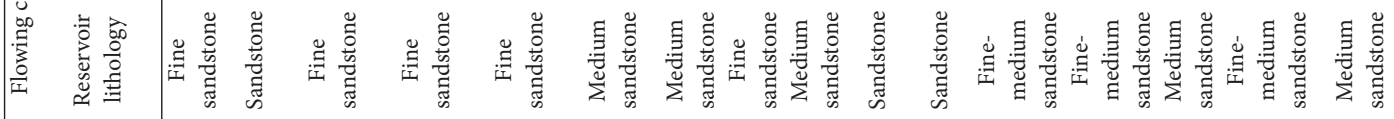

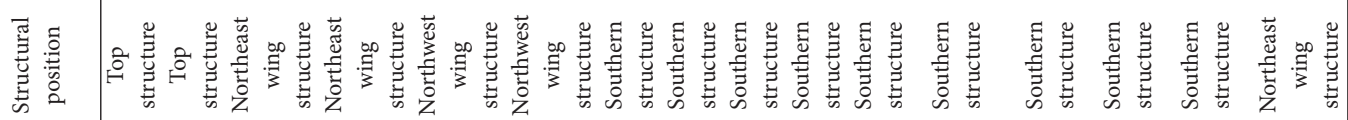

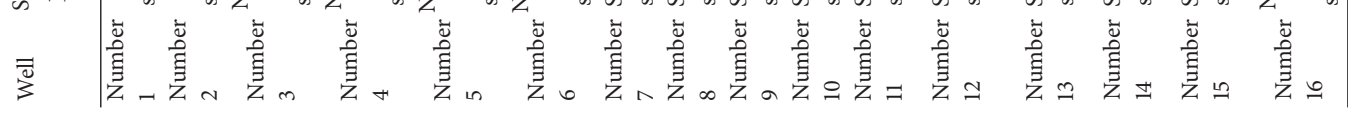


TABLE 4: Fracture decision system in reservoir capacity.

\begin{tabular}{|c|c|c|c|c|c|c|c|c|}
\hline Decision system & $\begin{array}{l}\text { Compensated } \\
\text { sonic } \\
\mu \mathrm{s} / \mathrm{ft}\end{array}$ & $\begin{array}{c}\text { Compensated } \\
\text { neutron } \\
\text { PU }\end{array}$ & $\begin{array}{c}\text { Compensated } \\
\text { density } \\
\mathrm{g} / \mathrm{cm}^{3}\end{array}$ & $\begin{array}{c}\text { Natural } \\
\text { gamma } \\
\text { API }\end{array}$ & $\begin{array}{c}\text { Net pay } \\
\text { thickness } \\
\text { m }\end{array}$ & Porosity \% & $\begin{array}{c}\text { Gas } \\
\text { saturation \% }\end{array}$ & $\begin{array}{c}\text { Oil-gas } \\
\text { display type }\end{array}$ \\
\hline I & $72-70$ & $18-15$ & $2.4-2.7$ & $61-66$ & $31-24$ & $12-9$ & $61-51$ & Well kick \\
\hline II & $70-68$ & $15-12$ & $2.7-2.9$ & $66-71$ & $24-18$ & $9-6$ & $5-41$ & Gas cut \\
\hline III & $68-66$ & $12-9$ & $2.9-3.1$ & $71-77$ & $18-12$ & $6-4$ & $41-30$ & $\begin{array}{c}\text { Gas logging } \\
\text { abnormal }\end{array}$ \\
\hline IV & $66-63$ & $9-6$ & $3.1-3.5$ & $77-82$ & $12-6$ & $4-2$ & $30-20$ & No display \\
\hline
\end{tabular}

TABLE 5: Fracture decision system in flow capacity.

\begin{tabular}{|c|c|c|c|c|}
\hline Decision system & Structural position & Reservoir lithology & Deep-shallow resistivity (OMM) & Mud density difference $\left(\mathrm{g} / \mathrm{cm}^{3}\right)$ \\
\hline I & Top structure & Fine sandstone & $2.39-1.39$ & $-0.09-(-0.02)$ \\
\hline II & Northeast wing structure & Sandstone & $1.39-0.39$ & $-0.02-0.04$ \\
\hline III & Northwest wing structure & Medium sandstone & $0.39-0.61$ & $0.04-0.11$ \\
\hline IV & Southern structure & Fine-medium sandstone & $-0.61-1.61$ & $0.11-0.17$ \\
\hline
\end{tabular}

TABLE 6: Fracture decision system in stimulation.

\begin{tabular}{lcccc}
\hline Decision system & Ahead fluid volume $\left(\mathrm{m}^{3}\right)$ & Sand-carrier volume $\left(\mathrm{m}^{3}\right)$ & Proppant volume $\left(\mathrm{m}^{3}\right)$ & Injection displacement $\left(\mathrm{m}^{3} / \mathrm{min}^{2}\right.$ \\
\hline I & $180-150$ & $190-170$ & $46-40$ & $3.1-3.2$ \\
II & $150-120$ & $170-150$ & $40-35$ & $3.2-3.3$ \\
III & $120-80$ & $150-130$ & $35-30$ & $3.3-3.4$ \\
IV & $80-50$ & $130-100$ & $30-25$ & $3.4-3.5$ \\
\hline
\end{tabular}

In this fractured gas reservoir, oil-gas display type, structural position, and reservoir lithology are discrete index. The oilgas display types are divided into well kick, gas cut, gas logging abnormal, and no display; structural position is divided into top structure, northeast wing structure, northwest wing structure, and construct the southern; reservoir lithology is divided into fine sandstone, fine-medium sandstone, medium sandstone, and sandstone. All the parametersof decision system are shown in Tables 4, 5, and 6 .

4.2. Establish Multiple Correlation Matrixes. According to the principle of multiple grey correlation, weights on the parameters of this natural gas field are divided into two levels; the calculation steps are as follows.
First, calculate the primary level weight vector $W 1$ of all the parameters through the single-level grey correlation calculation formula (8):

$W 1$

$$
\begin{aligned}
& =[0.0573,0.0689,0.0555,0.0518,0.0634, \\
& 0.0700,0.0700,0.0729,0.0709,0.0728 \text {, } \\
& 0.0671,0.0596,0.0549,0.0542,0.0552,0.0547] \text {. }
\end{aligned}
$$

Secondly, calculate the membership degree matrix $R 1$ though the membership function and the secondary membership degree matrix $R 2$ shown as follows:

$$
\begin{aligned}
R 2 & =W 1 \cdot R 1 \text {. } \\
& =\left[\begin{array}{cccc}
\text { Decision System } & \text { Reservoir Capacity } & \text { Folw Capacity } & \text { Stimulation } \\
\text { I } & 0.076 & 0.043 & 0.055 \\
\text { II } & 0.223 & 0.061 & 0.004 \\
\text { III } & 0.108 & 0.030 & 0 \\
\text { IV } & 0.060 & 0.086 & 0.068
\end{array}\right] .
\end{aligned}
$$


TABLE 7: Fuzzy comprehensive evaluation value and test production for all wells.

\begin{tabular}{|c|c|c|c|c|}
\hline Well & $\begin{array}{l}\text { Test production } \\
\left(10^{4} \mathrm{~m}^{3} / \mathrm{d}\right)\end{array}$ & $\begin{array}{c}\text { Fuzzy comprehensive } \\
\text { evaluation value }\end{array}$ & Reservoir level & $\begin{array}{c}\text { Development degree } \\
\text { of natural fractures }\end{array}$ \\
\hline Number 1 & 8.61 & 86 & $\mathrm{I}$ & Optimum \\
\hline Number 2 & 5.08 & 72 & II & Better \\
\hline Number 3 & 5.06 & 79 & II & Better \\
\hline Number 4 & 4.45 & 72 & II & Better \\
\hline Number 5 & 4.09 & 68 & II & Better \\
\hline Number 6 & 3.73 & 68 & II & Better \\
\hline Number 7 & 3.00 & 66 & II & Better \\
\hline Number 8 & 2.29 & 68 & II & Better \\
\hline Number 9 & 1.08 & 63 & III & Possible \\
\hline Number 10 & 1.00 & 64 & II & Better \\
\hline Number 11 & 0.95 & 60 & III & Possible \\
\hline Number 12 & 0.64 & 59 & III & Possible \\
\hline Number 13 & 0.57 & 57 & III & Possible \\
\hline Number 14 & 0.36 & 52 & III & Possible \\
\hline Number 15 & 0.12 & 35 & IV & None \\
\hline Number 16 & $?$ & 64.4 & II & Better \\
\hline
\end{tabular}

Finally, calculate secondary level weight vector $W 2$, through the multilevel grey correlation calculation formula (11), and get the gray correlation matrix $B 2$ by fuzzy arithmetic operator shown as follows:

$$
\begin{gathered}
W 2=\left[\begin{array}{lll}
0.5098, & 0.2704, & 0.2198
\end{array}\right] \\
B 2=W 2 \cdot R 2=\left[\begin{array}{llll}
0.062 & 0.131 & 0.063 & 0.068
\end{array}\right] .
\end{gathered}
$$

4.3. Quantitative Score. According to the gray correlation matrix the largest comprehensive evaluation correlation value is 0.131 , and the final fuzzy evaluation value is 64.4 through the calculation of function (5). The conclusion shows that the comprehensive evaluation of the well number 16 is closer to II level and the degree of reservoir fracture development in the medium:

$$
M=\sum_{j=1}^{n} v_{j} \times b_{j}=V \cdot B_{2}^{T}=64.4
$$

On the basis of single well reservoir evaluation, in order to forecast stimulation effect the author has carried on the quantitative score to all wells; Table 7 shows the test production after stimulation and fuzzy comprehensive evaluation value for all wells. As displayed in Table 7, different wells due to the different development degree of natural fractures and reservoir quality have different test production and reservoir comprehensive evaluation value. Compared with comprehensive evaluation value, well number 10 and well number 7 called similar wells are the closest two wells to the target well number 16 . Therefore, it concludes that the three wells reservoirs have a lot of similarities such as reservoir quality, fracture development degree, and stimulation test production. Then, it is predicted that the test production of well number $161.00 \times 10^{4}-3.00 \times 10^{4} \mathrm{~m}^{3} / \mathrm{d}$ between similar wells and also through score values the reservoir evaluation is with $80 \%$ accuracy.

4.4. Comparison of Fuzzy Logic with Other Methods. In comparison with the usefulness of these types of algorithms (fuzzy logic, fitting function, fuzzy clustering, BP neural network, and C4.5), it is needed to evaluate the adaptability to variety of methods based on the characteristics of reservoir evaluation.

Firstly, this study uses 16 parameters and their complicated nonlinear relationship exists between them, so simple parameter fitting function and fuzzy clustering are impossible to achieve high accuracy ( $80 \%$ compared to fuzzy logic).

Secondly, in reservoir evaluation process, it is necessary to know the weights indicating the influence extent of each parameter to the stimulation performance. BP neural network model is a black box with only inputs and outputs and C4.5 pruning through the maximum gain-ratio and they have a common characteristic that cannot reflect the middle of the process networks. Fuzzy logic algorithm can not only reflect the degree of membership for each parameter in each evaluation criteria system level, but also calculate the weight of each parameter affecting the gas well production.

Therefore, any single mathematical method is hard to describe the particularity of fractured gas well reservoir evaluation, but the method based on fuzzy logic and multiple grey correlation can be a good description of reservoir evaluation.

\section{Conclusion}

The economic success of a fractured gas reservoir depends on successful reservoir evaluation to find the reservoir with 
fracture development. Therefore accurate characterization parameter of natural fractures and mathematical model are required for reservoir evaluation and production prediction.

(1) Structural position, reservoir lithology, porositypermeability relationship, and drilling and logging display are effective parameters representing the degree of natural fracture development.

(2) The fuzzy logic and multiple grey correlation model is trained and tested with a case study in a Hechuan fractured gas reservoir. The proposed model is used accurately to have evaluated and ranked sixteen wells with four criteria. The results of the model were reasonable based on the facts that good wells are good reservoir quality for fracturing.

(3) The proposed system can provide outcomes to assist in fractured gas reservoir evaluation and ranking candidate wells for fracturing treatment

\section{Conflict of Interests}

The authors declare that there is no conflict of interests regarding the publication of this paper.

\section{Acknowledgments}

This work was supported by the Search Foundation of Sichuan Province under Grant no. 2014HH0004 and the National Natural Science Foundation of China (nos. 51134004 and 51374178). This work was also financially supported by 973 Program of China under Grant no. 2014CB239205.

\section{References}

[1] W. Narr, "Fracture density in the deep subsurface: techniques with application to Point Arguello oil field," American Association of Petroleum Geologists Bulletin, vol. 75, no. 8, pp. 13001323, 1991.

[2] C. L. Hanks, J. Lorenz, L. Teufel, and A. P. Krumhardt, "Lithologic and structural controls on natural fracture distribution and behavior within the Lisburne Group, northeastern Brooks Range and North Slope subsurface, Alaska," AAPG Bulletin, vol. 81, no. 10, pp. 1700-1720, 1997.

[3] J. Huang, D. V. Griffiths, and S.-W. Wong, "Characterizing natural-fracture permeability from mud-loss data," SPE Journal, vol. 16, no. 1, pp. 111-114, 2011.

[4] J. Norbeck, E. Fonseca, D. V. Griffiths, and S.-W. Wong, "Natural fracture identification and characterization while drilling underbalanced," in SPE Americas Unconventional Resources Conference, Pittsburgh, Pa, USA, 2012.

[5] M. J. Economides and T. Martin, Modern Fracturing: Enhancing Natural Gas Production, ET Publishing, 2007.

[6] M. Zoveidavianpoor, A. Samsuri et al., Development of a Fuzzy System Model for Candidate-Well Selection for Hydraulic Fracturing in a Carbonate Reservoir. SPE Oil and Gas India Conference and Exhibition, 2012.

[7] R. Nelson, Geologic Analysis of Naturally Fractured Reservoirs, Gulf Professional Publishing, 2001.
[8] J. C. Lorenz and S. P. Cooper, “Tectonic setting and characteristics of natural fractures in Mesaverde and Dakota reservoirs of the San Juan Basin," New Mexico Geology, vol. 25, no. 1, pp. 3-14, 2003.

[9] O. J. Ortega, J. F. W. Gale, and R. Marrett, "Quantifying diagenetic and stratigraphic controls on fracture intensity in platform carbonates: an example from the Sierra Madre Oriental, northeast Mexico," Journal of Structural Geology, vol. 32, no. 12, pp. 1943-1959, 2010.

[10] N. E. Odling, P. Gillespie, B. Bourgine et al., "Variations in fracture system geometry and their implications for fluid flow in fractured hydrocarbon reservoirs," Petroleum Geoscience, vol. 5, no. 4, pp. 373-384, 1999.

[11] J. E. Olson, S. E. Laubach, and R. H. Lander, "Natural fracture characterization in tight gas sandstones: integrating mechanics and diagenesis," AAPG Bulletin, vol. 93, no. 11, pp. 1535-1549, 2009.

[12] G. Chengjun, C. Kegui, and W. Yangan, Logging and Logging Evaluation Techniques for Carbonate Reservoir, Petroleum Industry Press, Beijing, China, 2007, (Chinese).

[13] L. Hunt, S. Reynolds, T. Brown, S. Hadley, J. Downton, and S. Chopra, "Quantitative estimate of fracture density variations in the Nordegg with azimuthal AVO and curvature: a case study," The Leading Edge, vol. 29, no. 9, pp. 1122-1137, 2010.

[14] R. Majidi, S. Z. Miska, M. Yu, L. G. Thompson, and J. Zhang, "Modeling of drilling fluid losses in naturally fractured formations," in SPE Annual Technical Conference and Exhibition, pp. 272-281, September 2008.

[15] L. A. Saputelli, M. Nikolaou, and M. J. Economides, "Selflearning reservoir management," SPE Reservoir Evaluation and Engineering, vol. 8, no. 6, pp. 534-547, 2005.

[16] J. Zhang, J. Li, Y. Hu, and J. Zhou, "The identification method of igneous rock lithology based on data mining technology," Applied Mechanics and Materials, vol. 466-467, pp. 65-69, 2012.

[17] S. Yu, K. Zhu, and F. Diao, "A dynamic all parameters adaptive BP neural networks model and its application on oil reservoir prediction," Applied Mathematics and Computation, vol. 195, no. 1, pp. 66-75, 2008.

[18] J. Finol, Y. Ke Guo, and X. D. Jing, "A rule based fuzzy model for the prediction of petrophysical rock parameters," Journal of Petroleum Science and Engineering, vol. 29, no. 2, pp. 97-113, 2001.

[19] H.-J. Zimmermann, "Fuzzy programming and linear programming with several objective functions," Fuzzy Sets and Systems, vol. 1, no. 1, pp. 45-55, 1978.

[20] R. Narasimhan, "Goal programming in a fuzzy environment," Decision Sciences, vol. 11, no. 2, pp. 325-336, 1980.

[21] D. Yin and T. Wu, "Optimizing well for fracturing by fuzzy analysis method of applying computer," in Proceedings of the 1st International Conference on Information Science and Engineering (ICISE '09), pp. 286-290, December 2009.

[22] D. Julong, "The grey control system," Journal of Huazhong University of Science and Technology, vol. 3, no. 9, article 18, 1982. 


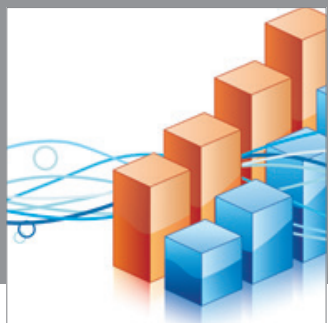

Advances in

Operations Research

mansans

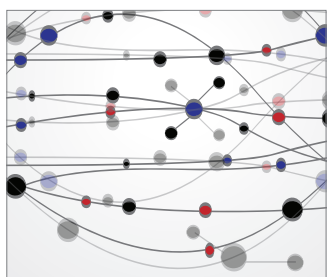

The Scientific World Journal
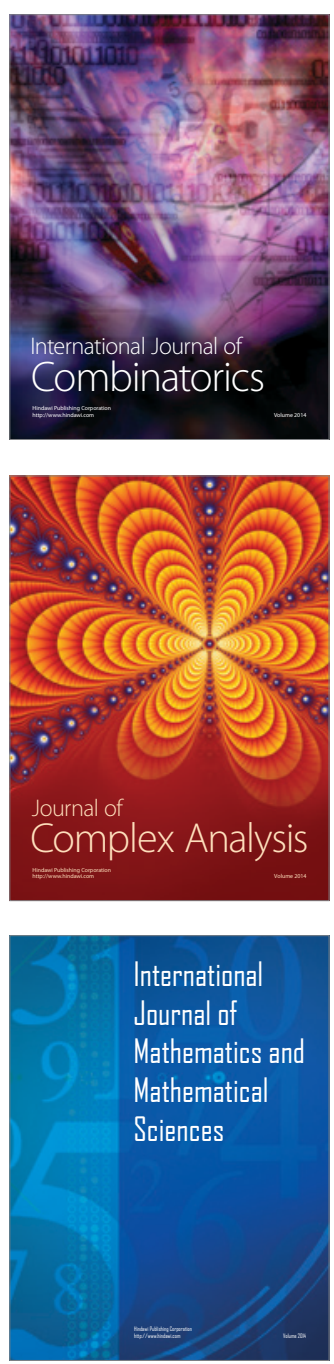
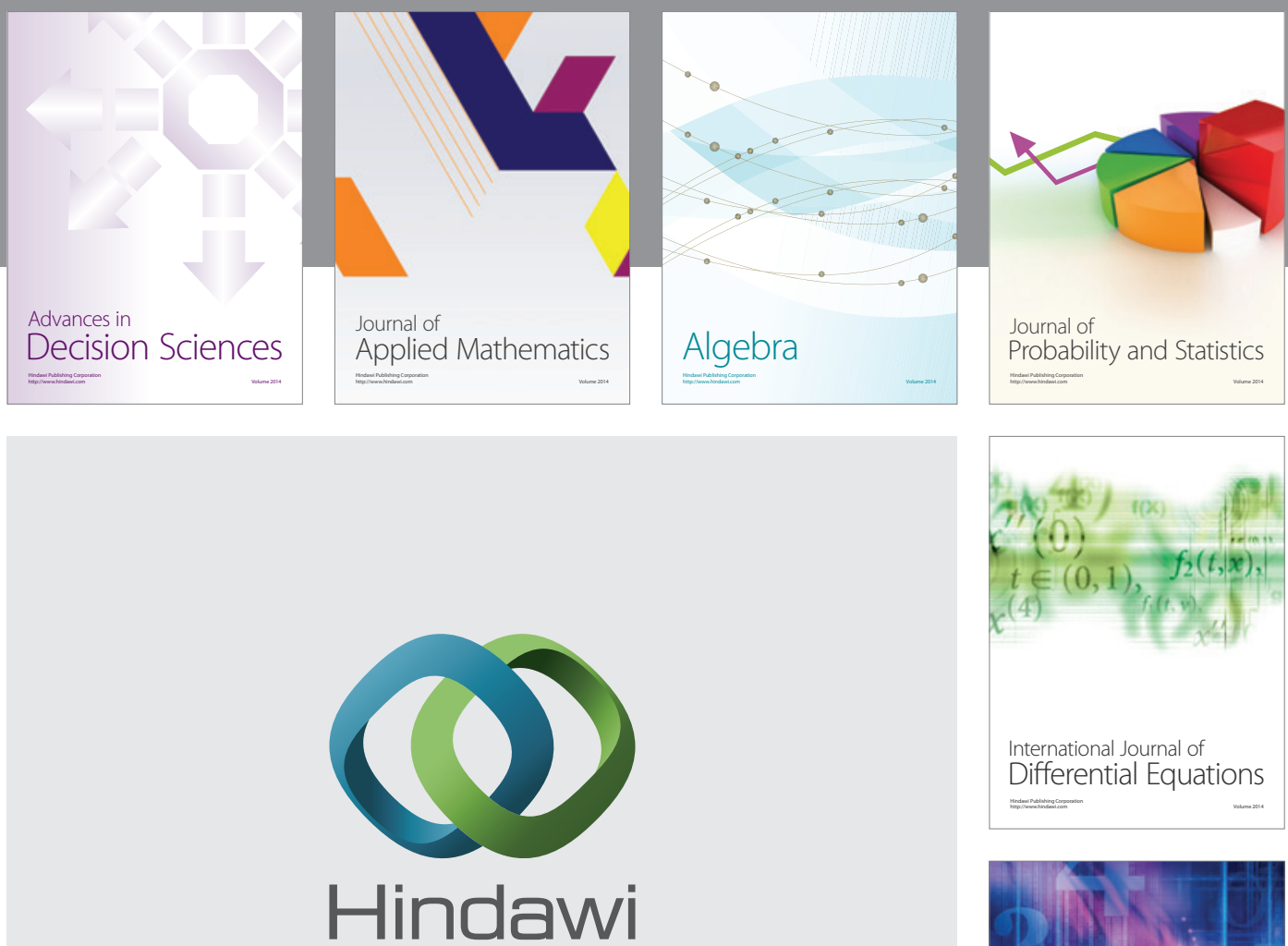

Submit your manuscripts at http://www.hindawi.com
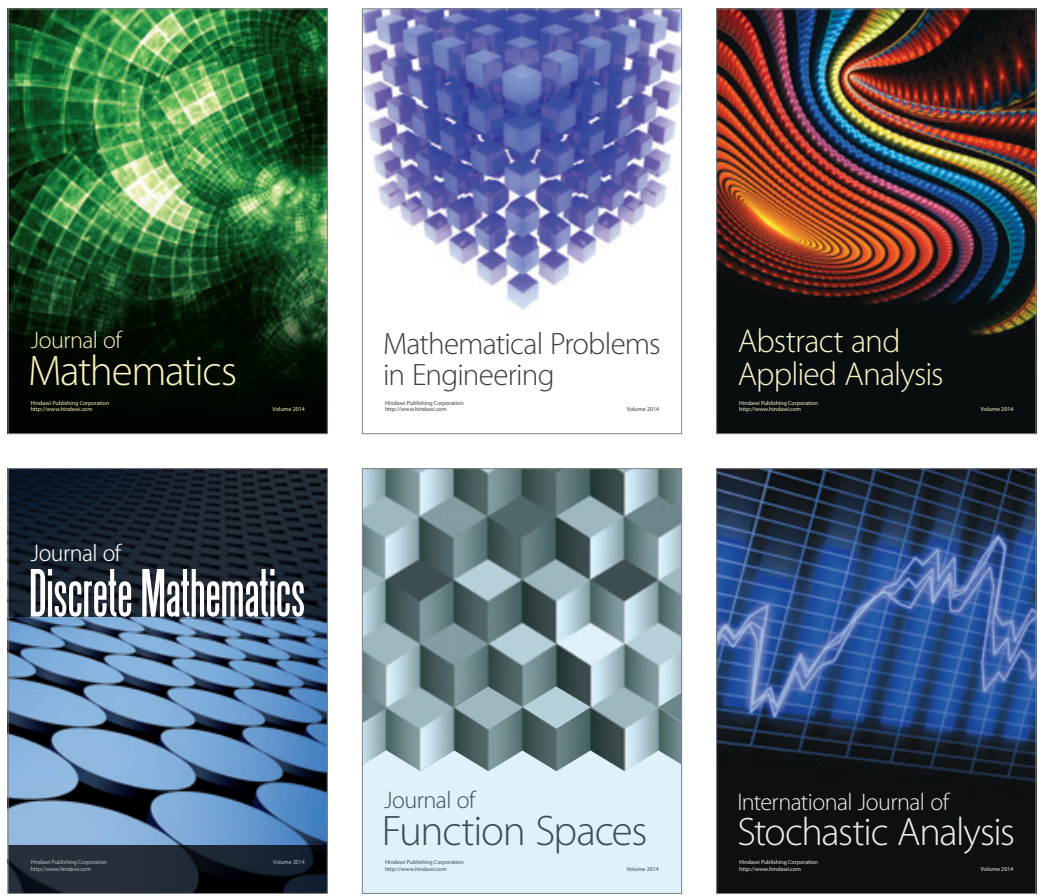

Journal of

Function Spaces

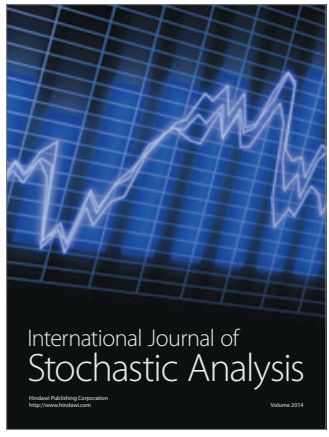

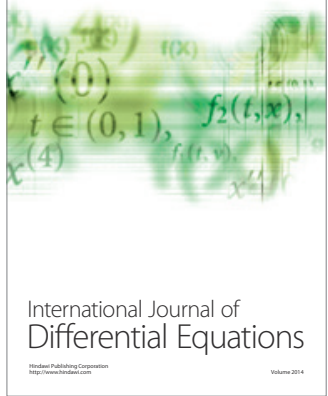
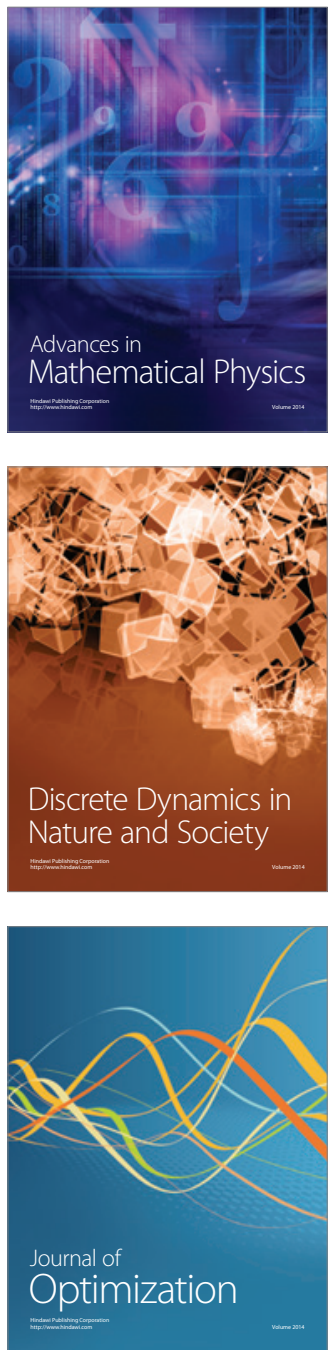